\title{
CONFORMAL HOLONOMY OF BI-INVARIANT METRICS
}

\author{
FELIPE LEITNER
}

\begin{abstract}
We discuss in this paper the conformal geometry of bi-invariant metrics on compact semisimple Lie groups. For this purpose, we develop an invariant Cartan calculus. Our main goal is to derive an iterative formula for the holonomy algebra of the normal conformal Cartan connection of a bi-invariant metric. As an example, we demonstrate the application of our invariant calculus to the computation of the conformal holonomy of $\mathrm{SO}(4)$. Its conformal holonomy algebra is $\mathfrak{s o}(7)$.
\end{abstract}

\section{INTRODUCTION}

In Riemannian geometry, the concept of holonomy is a well investigated topic. Holonomy groups arise from linear connections on Riemannian manifolds. Most famous is the holonomy theory of the Levi-Civita connection, which is the canonical connection to a Riemannian metric. A list of possible holonomy groups in this case was first established in 2. Also in conformal geometry certain connections can be introduced on the linear frame bundle of a space. Most notably there are the Weyl connections, which are torsion-free, taking values in $\mathfrak{c o}(s)$, the Lie algebra of the conformal structure group $\mathrm{CO}(s)$ of first order. However, a Weyl connection represents a priori an additional datum to a given conformal structure, and therefore, its holonomy cannot be seen as an invariant of the underlying conformal geometry.

Nevertheless, in conformal geometry (of dimension $s \geq 3$ ) there exits an invariant notion of holonomy. This holonomy can be naturally defined via the canonical Cartan connection of conformal geometry. The canonical Cartan connection lives on the prolongated principal fibre bundle of second order, whose structure group is a certain parabolic of the Möbius group $\mathrm{PO}(1, s+1$ ) (cf. e.g. 4] and [7). The canonical connection solves Cartan's equivalence problem for the case of conformal geometry, i.e., two conformal structures (on a common base space) are equivalent if and only if the canonical connections on the second order frame bundles are isomorphic. In general, the canonical connection takes values in the Lie algebra $\mathfrak{s o}(1, s+1)$ of the Möbius group, which implies that the conformal holonomy algebra is a Lie subalgebra of $\mathfrak{s o}(1, s+1)$. Similar as for the holonomy theory of Riemannian geometry, the conformal holonomy algebra and group are significant and useful invariants for a space with conformal structure. In particular, they describe invariant substructures and indicate solutions of certain overdetermined, conformally covariant partial differential equations (cf. [10, [12, 11], and [16]). Note that an alternative

Received by the editors April 19, 2007.

2000 Mathematics Subject Classification. Primary 53A30, 53C29; Secondary 53B15.

Key words and phrases. Conformal geometry, holonomy theory.

(C)2008 American Mathematical Society 
definition of the conformal holonomy can be achieved via tractor calculus and the celebrated tractor connection (cf. [5]).

The aim of this paper is to develop and apply an invariant Cartan calculus to a very simple situation, namely the conformal geometry of bi-invariant metrics on compact semisimple Lie groups, in order to compute conformal holonomy algebras (and groups) explicitly. We will proceed as follows. In the next two sections, we recall briefly the basic concept of conformal Cartan geometry, in general, and the well-known notion of bi-invariant metrics. In Section 4, we develop a conformal Cartan calculus adapted to bi-invariant metrics. In particular, we will describe the canonical connection and its curvature by certain linear maps $\gamma_{n o r}$ and $\kappa$. We discuss properties of these maps and derive a formula for the conformal holonomy algebra (cf. Section 5). Finally, we make explicit calculations for the bi-invariant metric on $\mathrm{SO}(4)$.

We note that a general discussion of invariant connections was recently developed in the wider context of homogeneous parabolic geometries in the works of M. Hammerl (cf. 14 and [15). These works apply the invariant calculus also to the special setting of homogeneous spaces in conformal and CR geometry. In particular, a nice relationship of the holonomy algebra and the algebra of infinitesimal automorphisms is established.

\section{Conformal Cartan geometry}

We briefly recall basic notions of canonical Cartan geometry on spaces with generic (Riemannian) conformal structure (cf. e.g. 4] or 7]).

Let $\mathbb{R}^{s}$ denote the euclidean space of dimension $s \geq 3$. We denote by $\mathfrak{g}$ the Lie algebra $\mathfrak{s o}(1, s+1)$ of the orthogonal group $\mathrm{O}(1, s+1)$, which acts via the standard representation on the Minkowski space $\mathbb{R}^{1, s+1}$ of dimension $s+2$ equipped with coordinates $\left(x_{-}, x_{1}, \ldots, x_{s}, x_{+}\right)$and invariant scalar product

$$
\langle x, y\rangle=x_{-} y_{+}+x_{+} y_{-}+\sum_{i=1}^{s} x_{i} y_{i} .
$$

The Lie algebra $\mathfrak{g}=\mathfrak{s o}(1, s+1)$ is $|1|$-graded by

$$
\mathfrak{g}=\mathfrak{m}_{-1} \oplus \mathfrak{g}_{0} \oplus \mathfrak{m}_{1}
$$

where $\mathfrak{g}_{0} \cong \mathfrak{c o}(s), \mathfrak{m}_{-1} \cong \mathbb{R}^{s}$ and $\mathfrak{m}_{1} \cong \mathbb{R}^{s *}$. We realise the subspaces $\mathfrak{g}_{0}, \mathfrak{m}_{-1}$ and $\mathfrak{m}_{1}$ of $\mathfrak{g}$ by matrices of the form

$$
\left(\begin{array}{ccc}
0 & 0 & 0 \\
m & 0 & 0 \\
0 & -m^{t} & 0
\end{array}\right) \in \mathfrak{m}_{-1}, \quad\left(\begin{array}{ccc}
-a & 0 & 0 \\
0 & A & 0 \\
0 & 0 & a
\end{array}\right) \in \mathfrak{g}_{0}, \quad\left(\begin{array}{ccc}
0 & l & 0 \\
0 & 0 & -l^{t} \\
0 & 0 & 0
\end{array}\right) \in \mathfrak{m}_{1} .
$$

The commutators are then given by

$$
\begin{array}{ll}
{[,]:} & \mathfrak{g}_{0} \times \mathfrak{g}_{0} \rightarrow \mathfrak{g}_{0}, \\
{[,]:} & \mathfrak{g}_{0} \times \mathfrak{m}_{-1} \rightarrow \mathfrak{m}_{-1}, \\
{[,]:} & \mathfrak{m}_{1} \times \mathfrak{g}_{0} \rightarrow \mathfrak{m}_{1}, \\
{[,]:} & \mathfrak{m}_{-1} \times \mathfrak{m}_{1} \rightarrow \mathfrak{g}_{0},
\end{array}
$$

$$
\begin{aligned}
& {\left[(A, a),\left(A^{\prime}, a^{\prime}\right)\right]=\left(A A^{\prime}-A^{\prime} A, 0\right),} \\
& {[(A, a), m]=A m+a m,} \\
& {[l,(A, a)]=l A+a l,} \\
& {[m, l]=\left(m l-(m l)^{t}, l m\right),}
\end{aligned}
$$

where $(A, a),\left(A^{\prime}, a^{\prime}\right) \in \mathfrak{s o}(s) \oplus \mathbb{R}, m \in \mathbb{R}^{s}, l \in \mathbb{R}^{s *}$. On $\mathfrak{m}_{-1} \cong \mathbb{R}^{s}$ we have the standard inner product $\langle\cdot, \cdot\rangle_{s}$, which is invariant under the action of the semisimple part $\mathfrak{s o}(s)$ of $\mathfrak{g}_{0}$. We denote the standard orthonormal basis on $\mathfrak{m}_{-1}$ by $\left\{e_{1}, \ldots, e_{s}\right\}$. 
The space

$$
\mathfrak{p}:=\mathfrak{g}_{0} \oplus \mathfrak{m}_{1}
$$

is a parabolic subalgebra of $\mathfrak{g}$. Whilst the grading of $\mathfrak{g}$ is not $\mathfrak{p}$-invariant, the filtration

$$
\begin{array}{lllll}
\mathfrak{g} & \supset & \mathfrak{p} & \supset \mathfrak{m}_{1}
\end{array}
$$

is $\mathfrak{p}$-invariant. Let $G:=\mathrm{PO}(1, s+1)$ denote the Möbius group, which is the projective orthogonal group $\mathrm{O}(1, s+1) / \mathbb{Z}_{2}$. The subgroup $G_{0}$ of $G$, which consists of those elements whose adjoint action on $\mathfrak{g}$ preserves the grading, is isomorphic to the reductive group $\mathrm{CO}(s)=\mathrm{O}(s) \times \mathbb{R}_{+}$with Lie algebra $\mathfrak{g}_{0}=\mathfrak{c o}(s)$. The parabolic subgroup $P$ of $G$ with Lie algebra $\mathfrak{p}$, which preserves the filtration of $\mathfrak{g}$, is then isomorphic to the semidirect product of $G_{0}$ and the vector subgroup $P_{1}:=\exp \left(\mathfrak{m}_{1}\right)$ (cf. e.g. 7]).

The $\mathfrak{g}_{0}$-homomorphism $\partial^{*}$ defined by

$$
\begin{aligned}
\partial^{*}: \operatorname{Hom}\left(\Lambda^{2} \mathfrak{m}_{-1}, \mathfrak{g}\right) & \rightarrow \operatorname{Hom}\left(\mathfrak{m}_{-1}, \mathfrak{g}\right), \\
\psi & \mapsto \partial^{*} \psi=\left(X \in \mathfrak{m}_{-1} \mapsto \sum_{i=1}^{s}\left[\eta_{i}, \psi\left(\xi_{i}, X\right)\right]\right),
\end{aligned}
$$

where $\left\{\xi_{i}: i=1, \ldots, s\right\}$ is some basis of $\mathfrak{m}_{-1}$ and $\left\{\eta_{i}: i=1, \ldots, s\right\}$ is the corresponding dual basis of $\mathfrak{m}_{1}$ (with respect to the Killing form $B_{\mathfrak{s o}(1, s+1)}$ ), is the so-called codifferential, which is adjoint to the differential $\partial$ computing the Lie algebra cohomology of $\mathfrak{m}_{-1}$ with values in $\mathfrak{g}$ (cf. e.g. [7]).

Now let $M^{s}$ be a $C^{\infty}$-manifold of dimension $s \geq 3$. Two smooth metrics $g$ and $\tilde{g}$ on $M$ are called conformally equivalent if there exists a $C^{\infty}$-function $\phi$ such that $\tilde{g}=e^{2 \phi} g$. A conformal structure $c$ on $M^{s}$ is by definition a class of conformally equivalent $C^{\infty}$-metrics. Let $G L(M)$ denote the general linear frame bundle on $M$. The choice of a conformal structure $c$ on $M$ is the same as a $G_{0}$-reduction $\mathcal{G}_{0}(M)$ of $G L(M)$. By the process of prolongation we obtain from $\mathcal{G}_{0}(M)$ the principal $P$ bundle $\mathcal{P}(M)$, which is a reduction of the second order linear frame bundle $G L^{2}(M)$ of $M$ (cf. e.g. 4] and [7]). The prolongation also gives rise to a canonical Cartan connection $\omega_{\text {nor }}$ on $\mathcal{P}(M)$ taking values in $\mathfrak{g}$, which is isomorphic as Lie algebra to $\mathbb{R}^{s} \oplus\left(\mathfrak{g}_{0} \ltimes \mathfrak{g}_{0}^{(1)}\right)$, where $\mathfrak{g}_{0}^{(1)}$ denotes the first prolongation of $\mathfrak{g}_{0}$ (cf. e.g. [4] and [7]).

In general, a Cartan connection $\omega$ on the principal $P$-bundle $\mathcal{P}(M)$ is a smooth 1-form with values in $\mathfrak{g}$ such that:

(1) $\omega\left(\chi_{A}\right)=A$ for all fundamental fields $\chi_{A}, A \in \mathfrak{p}$,

(2) $R_{g}^{*} \omega=A d\left(g^{-1}\right) \circ \omega$ for all $g \in P$ and

(3) $\left.\omega\right|_{T_{u} \mathcal{P}(M)}: T_{u} \mathcal{P}(M) \rightarrow \mathfrak{g}$ is a linear isomorphism for all $u \in \mathcal{P}(M)$.

The curvature 2 -form $\Omega$ of the Cartan connection $\omega$ is given by

$$
\Omega=d \omega+\frac{1}{2}[\omega, \omega] .
$$

This satisfies $\iota_{\chi_{A}} \Omega=0$ for all $A \in \mathfrak{p}$, i.e., the curvature $\Omega$ is trivial with respect to insertion of vertical vectors on $\mathcal{P}(M)$. The corresponding curvature function

$$
\kappa: \mathcal{P}(M) \rightarrow \operatorname{Hom}\left(\Lambda^{2} \mathfrak{m}_{-1}, \mathfrak{g}\right)
$$

is pointwise defined by

$$
\kappa(u)(X, Y):=d \omega\left(\omega_{u}^{-1}(X), \omega_{u}^{-1}(Y)\right)+[X, Y], \quad u \in \mathcal{P}(M), \quad X, Y \in \mathfrak{m}_{-1} .
$$

This function $\kappa$ decomposes to $\kappa_{-1}+\kappa_{0}+\kappa_{1}$ according to the grading of the space $\mathfrak{g}$. The map $\mathfrak{g}_{0} \rightarrow \mathfrak{g l}\left(\mathfrak{m}_{-1}\right)$ induced by the adjoint representation is the inclusion 
of a Lie subalgebra. Therefore, the 0-part $\kappa_{0}$ can be seen as a function on $\mathcal{P}(M)$, which takes values in $\mathfrak{m}_{-1}^{*} \otimes \mathfrak{m}_{-1}^{*} \otimes \mathfrak{m}_{-1}^{*} \otimes \mathfrak{m}_{-1}$.

The canonical connection $\omega_{\text {nor }}$ on $\mathcal{P}(M)$, naturally induced by the prolongation, satisfies the curvature normalisation

$$
\partial^{*} \kappa=0,
$$

and its $(-1)$-part $\omega_{-1}$ serves as the soldering form reproducing the given conformal structure $c$ on the base space $M$. These two properties determine $\omega_{\text {nor }}$ on $\mathcal{P}(M)$ uniquely. This is a basic fact of conformal geometry. (To be more accurate, $\omega_{\text {nor }}$ is uniquely determined up to an isomorphism between $\mathfrak{g}$ and the prolongation $\mathbb{R}^{s} \oplus$ $\left(\mathfrak{g}_{0} \ltimes \mathfrak{g}_{0}^{(1)}\right)$. Such an identification is obviously given via the grading $\mathfrak{m}_{-1} \oplus \mathfrak{g}_{0} \oplus \mathfrak{m}_{1}$ of $\mathfrak{g}$ and the identification $\mathfrak{m}_{-1} \cong \mathbb{R}^{s}$ as introduced above. Fixing this identification the canonical connection $\omega_{\text {nor }}$ is uniquely determined.) We call $\left(\mathcal{P}(M), \omega_{n o r}\right)$ the unique normal Cartan geometry over the base $M$ with conformal structure $c$. The condition $\partial^{*} \kappa=0$ is equivalent to

$$
\kappa_{-1}=0 \quad \text { and } \quad \operatorname{tr} \kappa_{0}:=\sum_{i=1}^{s} \kappa_{0}\left(\xi_{i}, \cdot\right)(\cdot)\left(\eta_{i}\right)=0,
$$

i.e., the unique normal Cartan connection $\omega_{\text {nor }}$ is torsion-free and the 0-part of the curvature function is traceless (cf. 4] and [7]). With respect to the choice of a Riemannian metric $g$ in the conformal class $c$ the content of the conformal curvature $\Omega\left(\right.$ resp. $\kappa=\kappa_{0}+\kappa_{1}$ ) is the Weyl curvature tensor $W^{g}$ and the Cotton tensor $C^{g}$.

\section{BI-INVARIANT METRICS}

We recall here the notion of a bi-invariant metric on a Lie group and do some curvature calculations in terms of Lie brackets (cf. e.g. [3]).

Let $N$ be a connected and compact semisimple Lie group of dimension $s$ and let $\mathfrak{n}$ denote its Lie algebra of left invariant vector fields. Then the Killing form

$$
B_{\mathfrak{n}}(X, Y):=\operatorname{trad}_{X} \operatorname{ad}_{Y}
$$

is an $\operatorname{Ad}(N)$-invariant negative definite scalar product on $\mathfrak{n}$. In particular,

$$
B_{\mathfrak{n}}(X,[Y, Z])=B_{\mathfrak{n}}([X, Y], Z) \quad \text { for all } X, Y, Z \in \mathfrak{n} .
$$

The Lie algebra $\mathfrak{n}$ is canonically identified with the tangent space $T_{e} N$ at the identity element. Thus the negative $-B_{\mathfrak{n}}$ of the Killing form gives rise to an inner product on $T_{e} N$, which by left translation with $L_{g}, g \in N$, extends uniquely to a smooth metric $g_{\mathfrak{n}}$ on $N$. Since $-B_{\mathfrak{n}}$ is $\operatorname{Ad}(N)$-invariant, right translation of $-B_{\mathfrak{n}}$ with $R_{g}$, $g \in N$, generates again $g_{\mathfrak{n}}$ and thus the metric $g_{\mathfrak{n}}$ on $N$ is called bi-invariant.

For the Levi-Civita connection $\nabla^{\mathfrak{n}}$ of the bi-invariant metric $g_{\mathfrak{n}}$ the equation

$$
\nabla_{X}^{\mathfrak{n}} Y=\frac{1}{2}[X, Y] \quad \text { for all } X, Y \in \mathfrak{n}
$$

holds. The Riemannian curvature tensor is then given by

$$
R_{X Y}^{\mathfrak{n}} Z=-\frac{1}{4}[[X, Y], Z] \quad \text { for all } X, Y, Z \in \mathfrak{n}
$$

and the sectional curvature of a plane spanned by orthonormal elements $X, Y$ in $\mathfrak{n}$ is

$$
S^{\mathfrak{n}}(X, Y):=\frac{1}{4} g_{\mathfrak{n}}([X, Y],[X, Y]) .
$$


For the Ricci tensor we have

$$
\operatorname{Ric}^{\mathfrak{n}}=\frac{1}{4} g_{\mathfrak{n}},
$$

i.e., $g_{\mathfrak{n}}$ is an Einstein metric on $N$ with positive scalar curvature $s c a l^{\mathfrak{n}}=\frac{s}{4}$. The Schouten tensor is $L^{\mathfrak{n}}=\frac{-1}{8(s-1)} g_{\mathfrak{n}}$ and the Cotton tensor $C^{\mathfrak{n}}$ vanishes identically. The Weyl tensor is given by

$$
W^{\mathfrak{n}}=R^{\mathfrak{n}}+\frac{1}{8(s-1)} g_{\mathfrak{n}} * g_{\mathfrak{n}},
$$

where $*$ denotes the Kulkarni-Nomizu product.

\section{INVARIANT CONFORMAL CARTAN CALCULUS FOR BI-INVARIANT METRICS}

At the end of the previous section we calculated the content of the conformal curvature (Weyl tensor and Cotton tensor) for a bi-invariant metric induced by the Killing form on a compact semisimple Lie group directly by use of the Lie bracket. In this section we establish an invariant conformal Cartan calculus for bi-invariant metrics on Lie groups. In particular, we will describe in this situation the normal Cartan connection and its curvature with respect to a certain linear map $\gamma_{\text {nor }}$. This approach will use a global trivialisation induced by a left invariant frame of second order, which stems from the invariant metric in the conformal class. We note that, although we assume a compact semisimple Lie group with bi-invariant metric coming from the Killing form, our discussion is actually valid for the more general situation of left invariant metrics on any Lie group. Only the explicit formulae for $\gamma_{n o r}$ and its curvature at the end of the section will depend on the bi-invariance.

Let $N$ be a connected and compact semisimple Lie group of dimension $s$ with Lie algebra $\mathfrak{n}$ and bi-invariant metric $g_{\mathfrak{n}}$. We fix the conformal structure $c_{\mathfrak{n}}=\left[g_{\mathfrak{n}}\right]$ on $N$. By definition, the Lie group $N$ acts by smooth isometries from the left on $\left(N, g_{\mathfrak{n}}\right)$, hence by conformal transformations on $\left(N, c_{\mathfrak{n}}\right)$. This action gives rise to induced left actions of $N$ on the first and second order conformal frame bundles $\mathcal{G}_{0}(N)$, resp., $\mathcal{P}(N)$. We denote all these actions by $L_{g}$ for $g \in N$. Obviously, the pullback $L_{g}^{*} \omega_{n o r}$ of the canonical connection by left translation with any $g \in N$ is again a Cartan connection on $\mathcal{P}(N)$. In fact, the $\mathfrak{m}_{-1}$-part of $L_{g}^{*} \omega_{n o r}$ reproduces the given conformal structure $c_{\mathfrak{n}}$ on $N$ and the curvature of $L_{g}^{*} \omega_{n o r}$ satisfies the normalisation condition $\partial^{*} \kappa=0$. Hence, it follows by the uniqueness of the normal Cartan connection (in the above sense) that

$$
L_{g}^{*} \omega_{n o r}=\omega_{n o r} \quad \text { for all } g \in N .
$$

Recall that $\mathfrak{m}_{-1} \cong \mathbb{R}^{s}$ is furnished with the standard inner product $\langle\cdot, \cdot\rangle_{s}$ and the standard orthonormal basis $\left\{e_{1}, \ldots, e_{s}\right\}$. Now let

$$
\theta:(\mathfrak{n},-B) \cong\left(\mathfrak{m}_{-1},\langle,\rangle_{s}\right)
$$

be an isometry of inner product spaces. We call $\theta$ a reference frame. It transfers the Lie bracket $[,]_{\mathfrak{n}}$ to $\mathfrak{m}_{-1}$ through the expression

$$
\rho_{\mathfrak{n}, \theta}(a, b):=\theta\left[\theta^{-1}(a), \theta^{-1}(b)\right]_{\mathfrak{n}},
$$

where $a, b \in \mathfrak{m}_{-1}$. Moreover, the reference frame $\theta$ induces the orthonormal frame

$$
\left\{E_{i}:=\theta^{-1}\left(e_{i}\right) \mid i=1, \ldots, s\right\}
$$


on $\mathfrak{n}$. The corresponding left-invariant orthonormal frame field on $N$ is a global trivialisation of the first order conformal frame bundle $\mathcal{G}_{0}(N)$ on $\left(N, c_{\mathfrak{n}}\right)$ :

$$
\begin{array}{cccc}
\mathcal{G}_{0}(N) & \cong & N \times \mathrm{CO}(s), \\
\left\{E_{i}(p) \mid i=1, \ldots, s\right\} & \mapsto & & (p, e) .
\end{array}
$$

(In fact, this is a trivialisation of the orthonormal frame bundle of $\left(N, g_{\mathfrak{n}}\right)$.) The bi-invariant metric $g_{\mathfrak{n}}$ induces a $C O(s)$-equivariant lift

$$
\sigma_{g_{\mathrm{n}}}: \mathcal{G}_{0}(N) \rightarrow \mathcal{P}(N)
$$

(i.e., a Weyl structure in the sense of [8]), which is also equivariant with respect to the induced left actions of $N$. Thus the composition of the left invariant frame $\left\{E_{i} \mid i=1, \ldots, s\right\}$ (as a section in $\mathcal{G}_{0}(N)$ ) with the lift $\sigma_{g_{\mathfrak{n}}}$ gives rise to a trivialisation

$$
\iota_{\theta, g_{\mathfrak{n}}}: \mathcal{P}(N) \cong N \times P
$$

of the second order frame bundle with parabolic structure group. As we have chosen the trivialisation $\iota_{\theta, g_{\mathfrak{n}}}$, the induced left action of $N$ on $\mathcal{P}(N)$ is given by

$$
\begin{aligned}
\iota_{\theta, g_{\mathfrak{n}}} \circ L_{g} \circ \iota_{\theta, g_{\mathfrak{n}}}^{-1}: N \times P & \rightarrow N \times P, \\
(n, p) & \rightarrow(g \cdot n, p) .
\end{aligned}
$$

The fact that the canonical Cartan connection $\omega_{n o r}$ is left invariant for the action of $N$ and right equivariant for the action of the parabolic structure group $P$ implies that $\omega_{\text {nor }}$ is uniquely determined by its values at a single point $x_{o} \in \mathcal{P}(N)$. We can choose $x_{o}$ as the point $(e, e)$ with respect to the trivialisation $\iota_{\theta, g_{\mathfrak{n}}}$ and then the linear map

$$
\omega_{\text {nor }}(e, e): \mathfrak{n} \times \mathfrak{p} \rightarrow \mathfrak{g}
$$

contains the whole information about $\omega_{\text {nor }}$ on $\mathcal{P}(N)$. We observe that the pullback of the reference frame $\theta$ to $\mathfrak{n} \times \mathfrak{p}$ equals the soldering form on $\mathcal{P}(N) \cong N \times P$ at $x_{o}$, i.e., the pullback of $\theta$ is identical to the (-1)-part of $\omega_{\text {nor }}$ at $x_{o} \in N \times P$. Thus we have $\omega_{-1}(e, e)\left(E_{i}\right)=e_{i}$ for any $i=1, \ldots, s$, where $E_{i}$ is to be seen as a vector in the first factor of $\mathfrak{n} \times \mathfrak{p}$. This leads us to the definition of the map $\gamma_{\text {nor }}$ (which depends on the choice of the global left invariant frame via the reference frame $\theta$ ) by

$$
\begin{aligned}
\gamma_{\text {nor }}: \mathfrak{m}_{-1} & \rightarrow \mathfrak{p}, \\
a & \mapsto \pi_{\mathfrak{p}} \circ \omega_{\text {nor }}(e, e) \circ \pi_{\mathfrak{n}} \circ \omega_{\text {nor }}^{-1}(e, e)(a),
\end{aligned}
$$

where $\pi_{\mathfrak{p}}$ and $\pi_{\mathfrak{n}}$ denote the obvious projections (the latter with respect to our trivialisation $\left.\iota_{\theta, g_{\mathfrak{n}}}\right)$. This map decomposes to

$$
\gamma_{\text {nor }}=\gamma_{0}+\gamma_{1}
$$

and still contains the whole information of the canonical Cartan connection $\omega_{\text {nor }}$ on $\mathcal{P}(N)$. In fact, via the relation

$$
\omega_{n o r}=\left(i d+\gamma_{n o r}\right) \circ \theta
$$

at $x_{o}=(e, e)$ the canonical Cartan connection $\omega_{\text {nor }}$ on $\mathcal{P}(N)$ can be recovered from $\gamma_{n o r}$. For the recovery, we simply extend $\left(i d+\gamma_{n o r}\right) \circ \theta$ at $x_{o}=(e, e)$ by $A d$-equivariant translation with $P$ and left invariant translation with $N$ to every point of $\mathcal{P}(N)$. 
The curvature $\Omega$ inherits the left and right invariance properties from the canonical connection $\omega_{\text {nor }}$ and $\Omega$ is also determined by its values at the single point $x_{o}=(e, e)$ in $P \times N$. With respect to the trivialisation $\iota_{\theta, g_{\mathfrak{n}}}$, we calculate

$$
\begin{aligned}
E_{i}\left(\omega_{n o r}\left(E_{j}\right)\right)(e, e) & =\left.\frac{d}{d t}\right|_{t=0} \omega_{n o r}\left(E_{j}\right)\left(\exp t E_{i}, e\right)=\left.\frac{d}{d t}\right|_{t=0} L_{\exp t E_{i}}^{*} \omega_{n o r}\left(E_{j}\right)(e, e) \\
& =\left.\frac{d}{d t}\right|_{t=0} \omega_{n o r}\left(E_{j}\right)(e, e)=0
\end{aligned}
$$

for all $i, j \in\{1, \ldots, s\}$. This shows the identity

$$
\Omega\left(E_{i}, E_{j}\right)=-\omega_{n o r}(e, e)\left(\left[E_{i}, E_{j}\right]_{\mathfrak{n}}\right)+\left[e_{i}+\gamma_{n o r}\left(e_{i}\right), e_{j}+\gamma_{n o r}\left(e_{j}\right)\right]_{\mathfrak{g}}
$$

The curvature function $\kappa$ of the canonical Cartan connection $\omega_{n o r}$ can then be expressed by (recall that the curvature is vertically trivial)

$$
\kappa\left(e_{i}, e_{j}\right)=-\left(i d+\gamma_{n o r}\right) \circ \rho_{\mathfrak{n}, \theta}\left(e_{i}, e_{j}\right)+\left[e_{i}+\gamma_{n o r}\left(e_{i}\right), e_{j}+\gamma_{n o r}\left(e_{j}\right)\right]_{\mathfrak{g}} .
$$

Thereby, the $(-1)$-part of $\kappa$ is given through

$$
\kappa_{-1}\left(e_{i}, e_{j}\right)=-\rho_{\mathfrak{n}, \theta}\left(e_{i}, e_{j}\right)+\left[e_{i}, \gamma_{0}\left(e_{j}\right)\right]_{\mathfrak{g}}+\left[\gamma_{0}\left(e_{i}\right), e_{j}\right]_{\mathfrak{g}} .
$$

This expression vanishes, since $\omega_{n o r}$ has no torsion, and we see that the Lie bracket of $\mathfrak{n}$ is given on $\mathfrak{m}_{-1}$ by

$$
\rho_{\mathfrak{n}, \theta}\left(e_{i}, e_{j}\right)=-\gamma_{0}\left(e_{j}\right) e_{i}+\gamma_{0}\left(e_{i}\right) e_{j}
$$

The 0 -part of $\kappa$ is

$\kappa_{0}\left(e_{i}, e_{j}\right)=-\gamma_{0} \circ \rho_{\mathfrak{n}, \theta}\left(e_{i}, e_{j}\right)+\left[e_{i}, \gamma_{1}\left(e_{j}\right)\right]_{\mathfrak{g}}+\left[\gamma_{1}\left(e_{i}\right), e_{j}\right]_{\mathfrak{g}}+\left[\gamma_{0}\left(e_{i}\right), \gamma_{0}\left(e_{j}\right)\right]_{\mathfrak{g}}$.

This part satisfies the trace-free condition

$$
\sum_{i=1}^{s} \gamma_{0} \circ \rho_{\mathfrak{n}, \theta}\left(e_{i}, a\right)(b)\left(e_{i}^{*}\right)=\left\{\begin{array}{c}
\sum_{i=1}^{s}\left[e_{i}, \gamma_{1}(a)\right]_{\mathfrak{g}}(b)\left(e_{i}^{*}\right)+\left[\gamma_{1}\left(e_{i}\right), a\right]_{\mathfrak{g}}(b)\left(e_{i}^{*}\right) \\
+\sum_{i=1}^{s}\left[\gamma_{0}\left(e_{i}\right), \gamma_{0}(a)\right]_{\mathfrak{g}}(b)\left(e_{i}^{*}\right)
\end{array}\right\}
$$

for all $a, b \in \mathfrak{m}_{-1}$, where $\left\{e_{i}^{*}: i=1, \ldots, s\right\}$ denotes the dual of the standard basis on $\mathfrak{m}_{-1}$. The 1-part $\kappa_{1}$ of the curvature is

$$
\kappa_{1}\left(e_{i}, e_{j}\right)=-\gamma_{1} \circ \rho_{\mathfrak{n}, \theta}\left(e_{i}, e_{j}\right)+\left[\gamma_{0}\left(e_{i}\right), \gamma_{1}\left(e_{j}\right)\right]_{\mathfrak{g}}+\left[\gamma_{1}\left(e_{i}\right), \gamma_{0}\left(e_{j}\right)\right]_{\mathfrak{g}}
$$

for all $i, j \in\{1, \ldots, s\}$.

The linear map $\gamma_{\text {nor }}: \mathfrak{m}_{-1} \rightarrow \mathfrak{p}$ is uniquely determined by the normalisation conditions (4.1) and (4.2) with respect to $\rho_{\mathfrak{n}, \theta}$. (Otherwise, we would recover from another map $\gamma$ subject to these properties via $\rho_{\mathfrak{n}, \theta}$ a further normal connection inducing $c_{\mathfrak{n}}$ on $N$, which is not possible.) This means that $\gamma_{n o r}$ depends only on the choice of $\theta$, which is used to transfer the Lie bracket of $\mathfrak{n}$ to $\mathfrak{m}_{-1}$. (To be more accurate again, the map $\gamma_{n o r}$ is only unique up to an identification of $\mathfrak{p}$ with the prolongation $\mathfrak{g}_{0} \ltimes \mathfrak{g}_{0}^{(1)}$.) We can introduce the following formal notions.

Definition 1. Let $(G, P)$ be the flat homogeneous model (of conformal geometry) with Lie algebras $(\mathfrak{g}, \mathfrak{h})$. Let $\langle\cdot, \cdot\rangle_{s}$ be the standard inner product on $\mathfrak{m}_{-1} \cong \mathbb{R}^{s}$ and let

$$
\rho: \mathfrak{m}_{-1} \times \mathfrak{m}_{-1} \rightarrow \mathfrak{m}_{-1}
$$

be a skew-symmetric map, which satisfies the Jacobi identity, and thus defines a Lie algebra bracket (of compact type) on the $(-1)$-part $\mathfrak{m}_{-1}$ of the grading of $\mathfrak{g}$. 
(1) We call a linear map

$$
\gamma=\gamma_{0}+\gamma_{1}: \mathfrak{m}_{-1} \rightarrow \mathfrak{p}
$$

an invariant connection form on $\mathfrak{m}_{-1}$ of type $(\mathfrak{g}, \mathfrak{p})$.

(2) The curvature

$$
\kappa_{\gamma, \rho}=\kappa_{-1}+\kappa_{0}+\kappa_{1}: \mathfrak{m}_{-1} \times \mathfrak{m}_{-1} \rightarrow \mathfrak{g}
$$

of the invariant connection $\gamma$ with respect to the Lie bracket $\rho$ is defined as

$$
\kappa_{\gamma, \rho}(a, b)=-(i d+\gamma) \circ \rho(a, b)+[(i d+\gamma)(a),(i d+\gamma)(b)]_{\mathfrak{g}}
$$

for $a, b \in \mathfrak{m}_{-1}$.

(3) The invariant connection $\gamma$ is called torsion-free with respect to $\rho$ if $\kappa_{-1}=0$.

(4) The invariant connection $\gamma$ is called normal with respect to $\rho$ if

$$
\kappa_{-1}=0 \quad \text { and } \quad \operatorname{tr} \kappa_{0}=0
$$

(cf. (4.2)).

(5) There exists a unique normal connection with respect to the bracket $\rho$ (and the standard choice $\langle\cdot, \cdot\rangle_{s}$ ), which we denote by $\gamma_{\rho}: \mathfrak{m}_{-1} \rightarrow \mathfrak{p}$ (or $\gamma_{\text {nor }}$ when the bracket is fixed on $\left.\mathfrak{m}_{-1}\right)$. We call $\gamma_{\rho}$ the canonical invariant connection form of type $(\mathfrak{g}, \mathfrak{p})$ corresponding to $\rho$ (and $\left.\langle\cdot, \cdot\rangle_{s}\right)$.

Note that (5) of Definition 1 is strictly speaking a lemma. To prove this lemma, we remark that any bracket $\rho$ on $\mathfrak{m}_{-1}$ is realised by a certain simply connected Lie group $N_{\rho}$ equipped with a left invariant metric via an identification $\theta: \mathfrak{n}_{\rho} \rightarrow$ $\left(\mathfrak{m}_{-1}, \rho,\langle\cdot, \cdot\rangle_{s}\right)$, which is an isometry and a Lie algebra isomorphism. The Lie group $N_{\rho}$, equipped with the conformal structure induced by the left invariant metric, admits a unique normal connection (in the sense as explained above), which induces $\gamma_{\rho}$ on $\mathfrak{m}_{-1}$ via $\theta$. The derived map $\gamma_{\rho}$ does not depend on the choice of $\theta$ !

As we can see from formula (4.1), the Lie bracket of $\mathfrak{n}$ is determined on $\mathfrak{m}_{-1}$ by the 0 -part $\gamma_{0}$ of the normal connection $\gamma_{n o r}$, since it has no torsion. In general, a linear map

$$
\gamma_{0}: \mathfrak{m}_{-1} \rightarrow \mathfrak{g}_{0},
$$

such that $-\gamma_{0}(b) \cdot a+\gamma_{0}(a) \cdot b$ is a skew-symmetric expression in $a, b \in \mathfrak{m}_{-1}$, defines a Lie bracket $\rho_{\gamma_{0}}(a, b)$ on $\mathfrak{m}_{-1}$ if and only if the following sum of even permutations vanishes for all $i, j, k \in\{1, \ldots, s\}$ (Jacobi identity):

$$
\sum_{\sigma(i, j, k)}\left[\gamma_{0}\left[e_{i}, \gamma_{0}\left(e_{j}\right)\right]+\gamma_{0}\left[\gamma_{0}\left(e_{i}\right), e_{j}\right]-\left[\gamma_{0}\left(e_{i}\right), \gamma_{0}\left(e_{j}\right)\right], e_{k}\right]=0 .
$$

The map $\gamma_{0}$ can then be extended in an arbitrary manner to a torsion-free connection form $\gamma$ with respect to $\rho_{\gamma_{0}}$ just by adding any linear 1-part $\gamma_{1}$. In such a situation, the curvature function to $\gamma$ with respect to $\rho_{\gamma_{0}}$ is given by

$$
\begin{aligned}
\kappa_{\gamma}(a, b)= & -\gamma_{0}\left(\left[a, \gamma_{0}(b)\right]+\left[\gamma_{0}(a), b\right]\right)+\left[a, \gamma_{1}(b)\right]+\left[\gamma_{1}(a), b\right]+\left[\gamma_{0}(a), \gamma_{0}(b)\right] \\
& -\gamma_{1}\left(\left[a, \gamma_{0}(b)\right]+\left[\gamma_{0}(a), b\right]\right)+\left[\gamma_{0}(a), \gamma_{1}(b)\right]+\left[\gamma_{1}(a), \gamma_{0}(b)\right] .
\end{aligned}
$$

Of course, not every torsion-free map $\gamma_{0}$ can be extended to the normal connection $\gamma_{\text {nor }}$ with respect to $\rho_{\gamma_{0}}$. The condition on $\gamma_{0}$ for being normally extendible is given 
by the existence of a $\gamma_{1}$ such that

$$
\begin{aligned}
\sum_{i=1}^{s}\left(\left[e_{i}, \gamma_{1}(a)\right]+\left[\gamma_{1}\left(e_{i}\right), a\right]\right)(b)\left(e_{i}^{*}\right) \\
\quad=\left\{\begin{array}{c}
\sum_{i=1}^{s} \gamma_{0}\left(\left[e_{i}, \gamma_{0}(a)\right]+\left[\gamma_{0}\left(e_{i}\right), a\right]\right)(b)\left(e_{i}^{*}\right) \\
-\sum_{i=1}^{s}\left[\gamma_{0}\left(e_{i}\right), \gamma_{0}(a)\right](b)\left(e_{i}^{*}\right)
\end{array}\right.
\end{aligned}
$$

for all $a, b \in \mathfrak{m}_{-1}$.

We note that so far in this section we have only made use of the left invariant nature of a metric on a Lie group, but not of the bi-invariant nature of $g_{\mathfrak{n}}$, which determines the conformal structure $c_{\mathfrak{n}}$. For the explicit calculation of $\gamma_{\text {nor }}$ and its curvature we specialise now to the case of a bi-invariant metric (induced by the Killing form) on a compact semisimple Lie group $N$. The map $\gamma_{0}$ corresponds in this situation to the Levi-Civita connection of the bi-invariant metric $g_{\mathfrak{n}}$ and is given with respect to the reference frame $\theta$ by

$$
\gamma_{0}\left(e_{i}\right)=\theta \circ \nabla_{E_{i}}^{\mathfrak{n}} \theta^{-1}(\cdot)=\frac{1}{2} \rho_{\mathfrak{n}, \theta}\left(e_{i}, \cdot\right)
$$

for all $i=1, \ldots, s$. Obviously, the so-defined map $\gamma_{0}$, considered as a matrix in $\mathfrak{g}_{0}=\mathfrak{c o}(s)$ with respect to the basis $\left\{e_{1}, \ldots, e_{s}\right\}$, satisfies (4.1), i.e., $\gamma_{0}$ is torsionfree with respect to $\mathfrak{n}$ (and $\theta$ ) (cf. Section 3 and [3]). Using (4.5) we calculate for the traces on the right-hand side of (4.4):

$$
\begin{aligned}
\sum_{i=1}^{s} \gamma_{0}\left(\left[e_{i}, \gamma_{0}(a)\right]+\left[\gamma_{0}\left(e_{i}\right), a\right]\right)(b)\left(e_{i}^{*}\right)= & \sum_{i=1}^{s} \gamma_{0}\left(\rho_{\mathfrak{n}, \theta}\left(e_{i}, a\right)\right)(b)\left(e_{i}^{*}\right) \\
= & \frac{1}{2} \sum_{i=1}^{s} \rho_{\mathfrak{n}, \theta}\left(\rho_{\mathfrak{n}, \theta}\left(e_{i}, a\right), b\right)\left(e_{i}^{*}\right) \\
= & \frac{1}{2} B_{\mathfrak{n}}\left(\theta^{-1}(a), \theta^{-1}(b)\right), \\
= & \frac{1}{4} \sum_{i=1}^{s} \rho_{\mathfrak{n}, \theta}\left(e_{i}, \rho_{\mathfrak{n}, \theta}(a, b)\right)\left(e_{i}^{*}\right) \\
& -\sum_{i=1}^{s} \rho_{\mathfrak{n}, \theta}\left(a, \rho_{\mathfrak{n}, \theta}\left(e_{i}, b\right)\right)\left(e_{i}^{*}\right) \\
= & \frac{1}{4} B_{\mathfrak{n}}\left(\theta^{-1}(a), \theta^{-1}(b)\right) .
\end{aligned}
$$

If we set $\gamma_{1}(a)=\lambda a^{*}$ for all $a \in \mathfrak{m}_{-1}$ with some constant $\lambda \in \mathbb{R}$, then calculation of the left-hand side of (4.4) results in

$$
\begin{aligned}
\sum_{i=1}^{s}\left(\left[e_{i}, \gamma_{1}\left(e_{k}\right)\right]+\left[\gamma_{1}\left(e_{i}\right), e_{k}\right]\right)\left(e_{l}\right)\left(e_{i}^{*}\right) \\
\quad=2 \lambda \sum_{i=1}^{s}\left(e_{i} \otimes e_{k}^{*}-e_{k} \otimes e_{i}^{*}\right)\left(e_{l}\right)\left(e_{i}^{*}\right) \\
\quad=2 \lambda(s-1) \delta_{k l}
\end{aligned}
$$

for all $k, l \in\{1, \ldots, s\}$. Comparing (4.6) and (4.7) shows that (4.4) is satisfied iff we set $\lambda:=\frac{-1}{8(s-1)}$, i.e., the normal connection form for $\mathfrak{n}$ (with bi-invariant metric) is determined to

$$
\begin{aligned}
\gamma_{\text {nor }}: \mathfrak{m}_{-1} & \rightarrow \mathfrak{p} \\
a & \mapsto \frac{1}{2} \rho_{\mathfrak{n}, \theta}(a, \cdot)-\frac{1}{8(s-1)} a^{*} .
\end{aligned}
$$

The curvature functions $\kappa_{-1}$ and $\kappa_{1}$ vanish identically, since there is no torsion and the Schouten tensor $L^{\mathfrak{n}}$ is covariantly constant with respect to $\nabla^{\mathfrak{n}}$. The latter fact 
implies that the Cotton tensor $C^{\mathfrak{n}}$ of $g_{\mathfrak{n}}$ vanishes. The 0-part $\kappa_{0}$ is given by the Weyl tensor $W^{\mathfrak{n}}$ of $g_{\mathfrak{n}}$, i.e., we have

$$
\kappa(a, b)=\kappa_{0}(a, b)=\theta \circ W^{\mathfrak{n}}\left(\theta^{-1}(a), \theta^{-1}(b)\right) .
$$

\section{InVARIANT CARTAN CONNECTION AND HOLONOMY}

In this section we briefly define the notion of conformal holonomy and establish a formula, which calculates the conformal holonomy algebra for the invariant connection $\gamma_{n o r}$ of the conformal class of a bi-invariant metric.

In general, let $\left(M^{s}, c\right), s \geq 3$, be a space with conformal structure $c$ and let $\mathcal{P}(M)$ be the principal $P$-bundle with canonical Cartan connection $\omega_{n o r}$. The bundle $\mathcal{P}(M)$ admits an extension to a principal $G$-bundle $\mathcal{G}(M)=\mathcal{P}(M) \times{ }_{P} G$, where $G=\mathrm{PO}(1, s+1)$ is the Möbius group. The canonical Cartan connection $\omega_{\text {nor }}$ extends by right translation to a principal bundle connection $\tilde{\omega}_{\text {nor }}$ on $\mathcal{G}(M)$ with (generic) structure group $G$. The extended principal connection gives rise to a holonomy group in the usual sense by means of parallel translation (cf. e.g. [3). This extension is a natural construction and thus gives rise to the notion of conformal holonomy group and algebra.

Definition 2. Let $(M, c)$ be a space with conformal structure $c$. The holonomy group $\mathrm{Hol}\left(\omega_{\text {nor }}\right)$ of the canonical Cartan connection $\omega_{\text {nor }}$ is defined to be the holonomy group of the naturally extended principal bundle connection $\tilde{\omega}_{\text {nor }}$ on $\mathcal{G}(M)$. The Lie algebra of $\operatorname{Hol}\left(\omega_{\text {nor }}\right)$ is denoted by $\mathfrak{h o l}\left(\omega_{\text {nor }}\right)$.

In general, the holonomy group $\mathrm{Hol}\left(\omega_{n o r}\right)$ is by definition a closed subgroup of $G=\operatorname{PO}(1, s+1)$ and $\mathfrak{h o l}\left(\omega_{n o r}\right)$ sits in $\mathfrak{s o}(1, s+1)$. We remark that there is a direct way to define the holonomy group of a Cartan connection without using the extended bundle (cf. [6]). The conformal holonomy group can also be defined via the canonical tractor connection (cf. [5]).

Now let $N$ be a connected and compact semisimple Lie group with Lie algebra $\mathfrak{n}$ and bi-invariant Riemannian metric $g_{\mathfrak{n}}$ coming from the Killing form and let $\gamma_{\text {nor }}: \mathfrak{m}_{-1} \cong \mathfrak{n} \rightarrow \mathfrak{p}$ be the invariant connection form corresponding to $\omega_{\text {nor }}$. We denote by

$$
\Lambda\left(\mathfrak{m}_{-1}\right):=\operatorname{span}\left\{\left(i d+\gamma_{\text {nor }}\right)(a) \mid a \in \mathfrak{m}_{-1}\right\} \subset \mathfrak{g}
$$

the image of the invariant connection and by

$$
\mathfrak{q}:=\operatorname{span}\left\{\kappa_{\mathfrak{n}}(a, b) \mid a, b \in \mathfrak{m}_{-1}\right\} \subset \mathfrak{p}
$$

the vector space of curvature values to $\gamma_{\text {nor }}$. There is a classical formula for the holonomy algebra of an invariant connection on a homogeneous space with arbitrary structure group $G$ (cf. [3]). This general result easily implies the following formula for the conformal holonomy algebra of a bi-invariant metric.

Theorem 5.1. Let $N$ be a connected and compact semisimple Lie group with conformal structure $\left[g_{\mathfrak{n}}\right]$. Then the holonomy algebra of the canonical Cartan connection $\omega_{\text {nor }}$ on $\left(N,\left[g_{\mathfrak{n}}\right]\right)$ is given by the iterative expression

$$
\mathfrak{h o l}\left(\omega_{n o r}\right)=\mathfrak{q}+[\Lambda(\mathfrak{n}), \mathfrak{q}]+[\Lambda(\mathfrak{n}),[\Lambda(\mathfrak{n}), \mathfrak{q}]]+\cdots,
$$

which is a Lie subalgebra of $\mathfrak{g}=\mathfrak{s o}(1, s+1)$. 


\section{EXAMPLES}

We make use of the invariant calculus for the conformal geometry of bi-invariant metrics in order to compute the conformal holonomy algebra in (a trivial and) a non-trivial case.

Example 1. Let $N=\mathrm{SO}(3)$ be the special orthogonal group in dimension 3, which is a 3-dimensional compact and semisimple Lie group. Let $\mathfrak{s o}(3)$ denote its Lie algebra. We introduce for $\mathfrak{s o}(3)$ the standard basis $\left\{E_{i j} \mid 1 \leq i<j \leq 3\right\}$, where the $E_{i j}$ 's are defined by matrix multiplication through

$$
E_{i j}:=e_{i} \cdot e_{j}^{t}-e_{j} \cdot e_{i}^{t}
$$

with respect to the standard basis $\left\{e_{1}, e_{2}, e_{3}\right\}$ of $\mathbb{R}^{3}$.

The Lie algebra $\mathfrak{s o}(3)$ is isomorphic to $\mathfrak{s u}(2)$ and the universal covering of the group $\mathrm{SO}(3)$ is

$$
S^{3}=\operatorname{Spin}(3) \cong \mathrm{SU}(2)
$$

The bi-invariant metric on $\mathrm{SO}(3)$ is conformally flat, since the Weyl tensor $W^{\mathfrak{n}}$ always vanishes in dimension 3 , and the Cotton $C^{\mathfrak{n}}$ vanishes for any bi-invariant metric. Of course, this is also clear from the fact that the bi-invariant metric on the universal covering group $\mathrm{SU}(2)$ is the standard metric on $S^{3}$. (Hence the calculation of conformal curvature and holonomy should produce trivial results.)

The connection form $\gamma_{\text {nor }}=\gamma_{0}+\gamma_{1}$ can be presented in the following form. The chosen basis $\left\{E_{i j}\right\}$ in $\mathfrak{s o}(3)$ is orthogonal and $-B_{\mathfrak{s o}(3)}\left(E_{i j}, E_{i j}\right)=2$ for all its elements. We define the reference frame

$$
\theta\left(\frac{1}{\sqrt{2}} E_{12}\right)=e_{1}, \quad \theta\left(\frac{1}{\sqrt{2}} E_{13}\right)=e_{2}, \quad \theta\left(\frac{1}{\sqrt{2}} E_{23}\right)=e_{3}
$$

Then we have

$$
\begin{aligned}
& \gamma_{0}\left(e_{1}\right)=\frac{1}{\sqrt{2}} \nabla_{E_{12}}^{\mathfrak{n}}=\frac{1}{2 \sqrt{2}}\left[E_{12}, \cdot\right]_{\mathfrak{n}}=\frac{1}{2 \sqrt{2}} E_{23}, \\
& \gamma_{0}\left(e_{2}\right)=\frac{1}{\sqrt{2}} \nabla_{E_{13}}^{\mathfrak{n}}=\frac{1}{2 \sqrt{2}}\left[E_{13}, \cdot\right]_{\mathfrak{n}}=-\frac{1}{2 \sqrt{2}} E_{13}, \\
& \gamma_{0}\left(e_{3}\right)=\frac{1}{\sqrt{2}} \nabla_{E_{23}}^{\mathfrak{n}}=\frac{1}{2 \sqrt{2}}\left[E_{23}, \cdot\right]_{\mathfrak{n}}=\frac{1}{2 \sqrt{2}} E_{12} .
\end{aligned}
$$

(Note for these formulae that the $E_{i j}$ 's form a basis of $\mathfrak{n}$ as well as a basis of the semisimple part of $\mathfrak{g}_{0}$.) For $\gamma_{1}$ we have

$$
\gamma_{1}\left(e_{i}\right)=-\frac{1}{16} e_{i}^{*}, \quad i=1,2,3
$$

These formulae express the normal Cartan connection on $\mathrm{SO}(3)$ in the trivialisation coming from the bi-invariant metric. One can easily see that the curvature $\kappa_{0}$ of $\gamma_{\text {nor }}$ vanishes identically. In particular, the conformal holonomy algebra is trivial, which results from the formula in Theorem 5.1 with $\mathfrak{q}=0$. 
Example 2. Now we apply the invariant Cartan calculus to the 6-dimensional compact and semisimple Lie group $\mathrm{SO}(4)$ with bi-invariant metric induced by the Killing form $B_{\mathfrak{s o}(4)}$. The Lie algebra $\mathfrak{s o}(4)$ is isomorphic to

$$
\mathfrak{s o}(3) \oplus \mathfrak{s o}(3)
$$

and as basis we use two copies of the basis $\left\{E_{i j}\right\}$ of $\mathfrak{s o}(3)$, namely

$$
\left\{E_{i j} \mid 1 \leq i<j \leq 3\right\} \cup\left\{E_{k l} \mid 4 \leq k<l \leq 6\right\} .
$$

This basis is orthogonal with $-B_{\mathfrak{s o}(4)}\left(E_{i j}, E_{i j}\right)=2$.

The bi-invariant metric $g_{\mathfrak{s o}(4)}$ induced by the Killing form is Einstein with positive scalar curvature. Obviously, it has non-constant sectional curvature (cf. the expression for $S^{\mathfrak{n}}$ in Section 31). Hence $\mathrm{SO}(4)$ with bi-invariant metric is not conformally flat. For that reason, we expect in our calculation non-trivial curvature terms and holonomy for the conformal structure $c_{\mathfrak{s o}(4)}$ on $\mathrm{SO}(4)$.

First, we calculate the normal connection form $\gamma_{\text {nor }}=\gamma_{0}+\gamma_{1}$. We use the reference frame

$$
\begin{aligned}
& \theta\left(\frac{1}{\sqrt{2}} E_{12}\right)=e_{1}, \quad \theta\left(\frac{1}{\sqrt{2}} E_{13}\right)=e_{2}, \quad \theta\left(\frac{1}{\sqrt{2}} E_{23}\right)=e_{3}, \\
& \theta\left(\frac{1}{\sqrt{2}} E_{45}\right)=e_{4}, \quad \theta\left(\frac{1}{\sqrt{2}} E_{46}\right)=e_{5}, \quad \theta\left(\frac{1}{\sqrt{2}} E_{56}\right)=e_{6} .
\end{aligned}
$$

From the calculations for the case of $\mathfrak{s o}(3)$ we know that

$$
\begin{array}{lll}
\gamma_{0}\left(e_{1}\right)=\frac{1}{2 \sqrt{2}} E_{23}, & \gamma_{0}\left(e_{2}\right)=-\frac{1}{2 \sqrt{2}} E_{13}, & \gamma_{0}\left(e_{3}\right)=\frac{1}{2 \sqrt{2}} E_{12}, \\
\gamma_{0}\left(e_{4}\right)=-\frac{1}{2 \sqrt{2}} E_{56}, & \gamma_{0}\left(e_{5}\right)=-\frac{1}{2 \sqrt{2}} E_{46}, & \gamma_{0}\left(e_{6}\right)=\frac{1}{2 \sqrt{2}} E_{45} .
\end{array}
$$

The 1-part $\gamma_{1}$ is given by

$$
\gamma_{1}\left(e_{i}\right)=-\frac{1}{40} e_{i}^{*}, \quad i=1, \ldots, 6 .
$$

We have to calculate the images of the 0-part $\kappa_{0}$ of the curvature function, which corresponds to the Weyl tensor

$$
W^{\mathfrak{n}}=R^{\mathfrak{n}}+\frac{1}{8(s-1)} g_{\mathfrak{n}} * g_{\mathfrak{n}},
$$

in order to obtain the space $\mathfrak{q}$. For the Kulkarni-Nomizu product in this expression for $W^{\mathfrak{n}}$, we easily see that

$$
\theta(B * B)\left(\theta^{-1}\left(e_{i}\right), \theta^{-1}\left(e_{j}\right)\right)=\frac{1}{20} E_{i j} \quad \text { for all } i<j \in\{1, \ldots, 6\} .
$$

For the Riemannian curvature tensor we find

$$
\theta R^{\mathfrak{n}}\left(\theta^{-1}\left(e_{i}\right), \theta^{-1}\left(e_{j}\right)\right)=-\frac{1}{8} E_{i j}
$$

for all $i<j \in\{1, \ldots, 3\}$ and all $i<j \in\{4, \ldots, 6\}$. The remaining curvature expressions for $R^{\mathfrak{n}}$ are zero. This shows that the span of the 0 -part $\kappa_{0}$ of the Cartan curvature is equal to $\mathfrak{s o}(6)$, which is the semisimple part of $\mathfrak{g}_{0}$ in the Möbius algebra $\mathfrak{s o}(1,7)$. Hence, we get for the span of the curvature values

$$
\mathfrak{q}=\mathfrak{s o}(6) \subset \mathfrak{g}_{0} .
$$

Obviously, the span of the normal connection $i d+\gamma_{\text {nor }}$ is given by

$$
\Lambda\left(\mathfrak{m}_{-1}\right)=\left\{e_{i}+\gamma_{0}\left(e_{i}\right)-\frac{1}{40} e_{i}^{*} \mid i=1, \ldots, 6\right\} .
$$


Then it is a straightforward calculation to see that the space $\left[\Lambda\left(\mathfrak{m}_{-1}\right), \mathfrak{q}\right]$ of commutators equals

We denote

$$
\operatorname{span}\left\{e_{i}-\frac{1}{40} e_{i}^{*} \mid i=1, \ldots, 6\right\} \oplus \mathfrak{s o}(6)
$$

$$
\mathfrak{l}:=\operatorname{span}\left\{x+\gamma_{1}(x) \mid x \in \mathfrak{m}_{-1}\right\} .
$$

The space $\mathfrak{l}$ is stable under the action of $\mathfrak{s o}(6)$ sitting in $\mathfrak{g}_{0}$. This shows that all the spaces

$$
\left[\Lambda\left(\mathfrak{m}_{-1}\right), \cdots,\left[\Lambda\left(\mathfrak{m}_{-1}\right), \mathfrak{q}\right] \cdots\right]
$$

of commutators are equal to $\mathfrak{l} \oplus \mathfrak{q}$, which is seen to be isomorphic to the Lie algebra $\mathfrak{s o}(7)$ embedded into $\mathfrak{s o}(1,7)$. We conclude for the holonomy algebra of $\mathrm{SO}(4)$ that

$$
\mathfrak{h o l}\left(\omega_{\text {nor }}\right)=\mathfrak{l} \oplus \mathfrak{q} \cong \mathfrak{s o}(7) .
$$

This result has the following interpretation. The bi-invariant metric $g_{\mathfrak{n}}$ in the conformal class $c_{\mathfrak{n}}$ on $N=\mathrm{SO}(4)$ is Einstein. It is known in general that the conformal Einstein condition implies that the holonomy algebra $\mathfrak{h o l}\left(\omega_{\text {nor }}\right)$ of the normal Cartan connection annihilates a standard tractor, that is a vector in the standard representation space $\mathbb{R}^{1,7}$ of the Lie algebra $\mathfrak{s o}(1,7)$ (cf. [1, [5], [10, and [1]). If the scalar curvature of the Einstein metric is positive, this vector is timelike, which explains that for our case the holonomy algebra hol $\left(\omega_{\text {nor }}\right)$ of $\mathrm{SO}(4)$ is automatically reduced to $\mathfrak{s o}(7)$. Our calculation then shows that the holonomy is not further reduced and we conclude that up to constant scales the bi-invariant metric is the only Einstein metric in its conformal class on $\mathrm{SO}(4)$. Moreover, we can deduce from the holonomy result that $\mathrm{SO}(4)$ does not admit twistor spinors nor conformal Killing forms satisfying a certain normalisation condition (cf. 9 ] and [11). Finally, we can say that $\mathrm{SO}(4)$ is not (locally) conformally equivalent to a product of Einstein metrics in such a manner that the Schouten tensor of the product equals the product of the Schouten tensors of the factors (cf. [11] and [13]).

\section{ACKNOWLEDGEMEnTS}

I would like to thank the referee whose comments and remarks certainly exerted a positive influence on the final form of this paper.

\section{REFERENCES}

1. S. Sasaki. On the spaces with normal conformal connexions whose groups of holonomy fix a point or a hypersphere. Jap. J. Math. 18, (1943).

2. M. Berger. Sur les groupes d'holonomie homogene des varietes a connexion affine et des varietes riemanniennes, Bull. Soc. Math. France 83(1955), p. 279-330. MR0079806 (18:149a)

3. S. Kobayashi, K. Nomizu. Foundations of differential geometry I \& II, John Wiley \& Sons, New York, 1963/69.

4. S. Kobayashi. Transformation Groups in Differential Geometry. Springer-Verlag Berlin Heidelberg, 1972. MR0355886(50:8360)

5. T.N. Bailey, M. Eastwood, A.R. Gover. Thomas's structure bundle for conformal, projective and related structures. Rocky Mountain J. Math. 24 (1994), no. 4, 1191-1217. MR1322223 (96e:53016)

6. R.W. Sharpe. Differential Geometry, Graduate Texts in Mathematics 166. Springer-Verlag New York, 1997. MR:1453120 (98m:53033)

7. A. Čap, J. Slovák, V. Souček. Invariant Operators on Manifolds with Almost Hermitian Symmetric Structures I \& II. Acta. Math. Univ. Comen., New Ser. 66, No. 1, p. 33-69 \& No. 2, p. 203-220(1997). 
8. A. Čap, J. Slovák. Weyl structures for parabolic geometries. Math. Scand. 93, 1 (2003) 53-90, electronically available as ESI Preprint 801. MR.1997873 (2004j:53065)

9. F. Leitner. Normal conformal Killing forms. e-print: arXiv:math.DG/0406316 (2004).

10. S. Armstrong. Definite signature conformal holonomy: a complete classification. e-print: arXiv:math/0503388 (2005). MR2348277

11. F. Leitner. Conformal Killing forms with normalisation condition. Rend. Circ. Mat. Palermo (2) Suppl. No. 75 (2005), 279-292. e-print: arXiv:math/0406316 (2004). MR2152367 (2006d:53029)

12. A. Cap, A.R. Gover. A holonomy characterisation of Fefferman spaces. e-print: arXiv:math/ 0611939 (2006).

13. A.R. Gover, F. Leitner. A sub-product construction of Poincaré-Einstein metrics. arXiv: math/0608044 (2006).

14. M. Hammerl. Homogeneous Cartan Geometries (master thesis). http://www.mat.univie. ac.at/ cap/files/Hammerl.pdf, 2006.

15. M. Hammerl. Homogeneous Cartan Geometries. e-print: arXiv:math/0703627 (2007).

16. Felipe Leitner, A remark on unitary conformal holonomy, IMA Volumes in Mathematics and its Applications: Symmetries and Overdetermined Systems of Partial Differential Equations, Editors: Michael Eastwood and Willard Miller, Jr., Springer New York, Volume 144 (2007), p. $445-461$.

Institut für Geometrie und Topologie, Universität Stuttgart, Pfaffenwaldring 57, Stuttgart-Vaihingen, D-70569, Germany

E-mail address: leitner@mathematik.uni-stuttgart.de 